\title{
Variational approach to non-instantaneous impulsive nonlinear differential equations
}

\author{
Liang Baia, ${ }^{a, *}$ Juan J. Nieto ${ }^{\mathrm{b}}$, Xiaoyun Wang ${ }^{\mathrm{a}}$ \\ ${ }^{a}$ College of Mathematics, Taiyuan University of Technology, Taiyuan, Shanxi 030024, P. R. China. \\ ${ }^{b}$ Departamento de Estadística, Análisis Matemático y Optimización, Facultad de Matemáticas, Universidad de Santiago de \\ Compostela, Santiago de Compostela 15782, Spain.
}

Communicated by X.-J. Yang

\begin{abstract}
In this paper, a class of nonlinear differential equations with non-instantaneous impulses are considered. By using variational methods and critical point theory, a criterion is obtained to guarantee that the non-instantaneous impulsive problem has at least two distinct nonzero bounded weak solutions. (C)2017 All rights reserved.
\end{abstract}

Keywords: Non-instantaneous impulse, mountain pass theorem, bounded solution.

2010 MSC: 34K45, 34B37, 47J30.

\section{Introduction}

Non-instantaneous impulsive differential equations were introduced by Hernádez and O'Regan in [7], motivated by a problem related to the hemodynamical equilibrium of a person: in the case of a decompensation (for example, high or low levels of glucose) one can prescribe some intravenous drugs (insulin). Since the introduction of the drugs in the bloodstream and the consequent absorption for the body are gradual and continuous processes, we can interpret the above situation as an impulsive action which starts abruptly and stays active on a finite time interval.

Impulsive effects arise from the real world and are used to describe sudden, discontinuous jumps. Differential equation with not instantaneous impulses is a generalization of the classical theory of impulsive differential equations. For some general and recent works on the theory of impulsive differential equations we refer the readers to $[1,2,4,6,9,10,13-16]$.

The existence of solutions of non-instantaneous impulsive problem has been studied via some approaches, such as fixed point theory and theory of analytic semigroup, see, for example, [5, 7, 11, 12]. Recently, the variational structure of non-instantaneous impulsive linear problem has been developed in [3].

\footnotetext{
*Corresponding author

Email addresses: tj_bailiang@126.com (Liang Bai), juanjose.nieto.roig@usc.es (Juan J. Nieto), wangxiaoyun@tyut.edu.cn (Xiaoyun Wang)
}

doi:10.22436/jnsa.010.05.14 
Inspired by the above facts, in this paper a class of non-instantaneous impulsive nonlinear problems which has variational structure will be studied by critical point theory.

Consider the following non-instantaneous impulsive problem

$$
\begin{cases}-u^{\prime \prime}(t)=D_{x} F_{i}\left(t, u(t)-u\left(t_{i+1}\right)\right), & t \in\left(s_{i}, t_{i+1}\right], i=0,1, \cdots, N, \\ u^{\prime}(t)=\alpha_{i}, & t \in\left(t_{i}, s_{i}\right], i=1,2, \cdots, N, \\ u^{\prime}\left(s_{i}^{+}\right)=u^{\prime}\left(s_{i}^{-}\right), & i=1,2, \cdots, N, \\ u(0)=u(T)=0, & u^{\prime}(0)=\alpha_{0},\end{cases}
$$

where $0=s_{0}<t_{1}<s_{1}<t_{2}<s_{2}<\cdots<t_{N}<s_{N}<t_{N+1}=T$, the impulses start abruptly at the points $t_{i}$ and keep the derivative constant on a finite time interval $\left(t_{i}, s_{i}\right]$. Here $u^{\prime}\left(s_{i}^{ \pm}\right)=\lim _{s \rightarrow s_{i}^{ \pm}} u^{\prime}(s)$ and $\alpha_{i}$ are given constants. For each $i=0,1, \cdots, N$, the nonlinear functions $D_{x} F_{i}(t, x)$ are the derivatives of $F_{i}(t, x)$ with respect to $x$ and $F_{i}$ satisfies the following assumption:

(A) $F_{i}(t, x)$ is measurable in $t$ for every $x \in \mathbb{R}$ and continuously differentiable in $x$ for a.e. $t \in\left(s_{i}, t_{i+1}\right]$, and there exist $a \in C\left(\mathbb{R}^{+} ; \mathbb{R}^{+}\right)$and $b \in \mathrm{L}^{1}\left(s_{i}, t_{i+1} ; \mathbb{R}^{+}\right)$such that

$$
\left|F_{i}(t, x)\right| \leqslant a(|x|) b(t), \quad\left|D_{x} F_{i}(t, x)\right| \leqslant a(|x|) b(t)
$$

for all $x \in \mathbb{R}$ and a.e. $t \in\left(s_{i}, t_{i+1}\right]$.

Moreover, without loss of generality, it is supposed that $F_{i}(t, 0)=0$ for a.e. $t \in\left(s_{i}, t_{i+1}\right]$ and each $i=0,1, \cdots, N$.

Our results are presented as follows.

Theorem 1.1. Assume condition (A) holds and

(H) for each $i=0,1, \cdots, N$, there exist constants $\sigma_{i}>2$ such that $0<\sigma_{i} F_{i}(t, x) \leqslant x D_{x} F_{i}(t, x)$ for a.e. $\mathrm{t} \in\left(\mathrm{s}_{\mathrm{i}}, \mathrm{t}_{\mathrm{i}+1}\right]$ and $\mathrm{x} \in \mathbb{R} \backslash\{0\}$,

then problem (1.1) has at least one nonzero bounded weak solution in $\mathrm{H}_{0}^{1}(0, \mathrm{~T})$ provided

$$
\frac{\pi^{2}}{4 T(1+\pi)^{2}}>\sum_{i=1}^{N}\left|\alpha_{i-1}-\alpha_{i}\right|+2 \sum_{i=0}^{N} \int_{s_{i}}^{t_{i+1}} \max _{|x|=1} F_{i}(t, x) d t .
$$

In addition, if $\alpha_{j-1} \neq \alpha_{j}$ for some $\mathrm{j}=1,2, \cdots, \mathrm{N}$, then problem (1.1) has at least two distinct nonzero bounded weak solutions in $\mathrm{H}_{0}^{1}(0, \mathrm{~T})$.

Example 1.2. It follows from Theorem 1.1 that the following non-instantaneous impulsive problem has at least two distinct nonzero bounded weak solutions.

$$
\left\{\begin{array}{l}
-u^{\prime \prime}(t)=t\left(u(t)-u\left(t_{i+1}\right)\right)^{3}, \quad t \in\left(s_{i}, t_{i+1}\right], i=0,1 \\
u^{\prime}(t)=0.1, \quad t \in\left(t_{1}, s_{1}\right] \\
u^{\prime}\left(s_{1}^{+}\right)=u^{\prime}\left(s_{1}^{-}\right), \\
u(0)=u(1)=0, \quad u^{\prime}(0)=0
\end{array}\right.
$$

where $0=s_{0}<t_{1}=\frac{1}{16}<s_{1}=\frac{15}{16}<t_{2}=1$.

\section{Preliminaries}

We recall some facts which will be used in the proof of our main result. It is a consequence of Poincaré's inequality that

$$
\int_{0}^{T}|u(t)|^{2} d t \leqslant \frac{1}{\lambda_{1}} \int_{0}^{T}\left|u^{\prime}(t)\right|^{2} d t
$$


where $\lambda_{1}=\pi^{2} / \mathrm{T}^{2}$ is the first eigenvalue of the Dirichlet problem

$$
-u^{\prime \prime}(t)=\lambda u(t), t \in[0, T] ; \quad u(0)=u(T)=0 .
$$

Let $\mathrm{H}_{0}^{1}(0, \mathrm{~T})$ be the Sobolev space endowed with the norm

$$
\|\mathrm{u}\|_{\mathrm{H}_{0}^{1}}:=\left(\int_{0}^{\mathrm{T}}\left|\mathrm{u}^{\prime}(\mathrm{t})\right|^{2}+|\mathrm{u}(\mathrm{t})|^{2} \mathrm{dt}\right)^{1 / 2} .
$$

Obviously, $\mathrm{H}_{0}^{1}(0, T)$ is a reflexive Banach space and by the Poincaré's inequality (2.1), we know that

$$
\|\mathrm{u}\|:=\left(\int_{0}^{T}\left|\mathrm{u}^{\prime}(\mathrm{t})\right|^{2} \mathrm{dt}\right)^{1 / 2}
$$

is equivalent to the norm $\|\mathrm{u}\|_{\mathrm{H}_{0}^{1}}$ in $\mathrm{H}_{0}^{1}(0, \mathrm{~T})$. Let

$$
\|\mathrm{u}\|_{\mathrm{L}^{2}}:=\left(\int_{0}^{T}|\mathrm{u}(\mathrm{t})|^{2} \mathrm{dt}\right)^{1 / 2} \text { and }\|\mathrm{u}\|_{\infty}:=\max _{\mathrm{t} \in[0, \mathrm{~T}]}|\mathrm{u}(\mathrm{t})| .
$$

Then for $u \in H_{0}^{1}(0, T)$, we have

$$
\|u\|_{\infty} \leqslant \beta\|u\|,
$$

where $\beta=\left(T \lambda_{1}\right)^{-1 / 2}+T^{1 / 2}$. In fact, it follows from the mean value theorem that $\frac{1}{T} \int_{0}^{T} u(s) d s=u(\tau)$ for some $\tau \in(0, T)$. Hence, for $t \in[0, T]$, using Hölder inequality,

$$
|u(t)|=\left|u(\tau)+\int_{\tau}^{t} u^{\prime}(s) d s\right| \leqslant \frac{1}{T}\left|\int_{0}^{T} u(s) d s\right|+\int_{0}^{T}\left|u^{\prime}(t)\right| d t \leqslant T^{-1 / 2}\|u\|_{L^{2}}+T^{1 / 2}\left\|u^{\prime}\right\|_{L^{2}}
$$

Lemma 2.1 ([17, Theorem 38.A]). For the functional $\mathrm{F}: M \subseteq X \rightarrow[-\infty,+\infty]$ with $M \neq \emptyset, \min _{\mathfrak{u} \in M} \mathrm{~F}(\mathrm{u})=\alpha$ has a solution in case the following hold:

(i) $X$ is a real reflexive Banach space;

(ii) $\mathrm{M}$ is bounded and weak sequentially closed;

(iii) $\mathrm{F}$ is sequentially weakly lower semi-continuous on $\mathrm{M}$.

Lemma 2.2 ([8, Theorem 4.10]). Let $\mathrm{E}$ be a Banach space and $\varphi \in \mathrm{C}^{1}(\mathrm{E}, \mathbb{R})$. Assume that there exist $\mathrm{u}_{0} \in \mathrm{E}$, $\mathrm{u}_{1} \in \mathrm{E}$ and $a$ bounded open neighborhood $\Omega$ of $\mathfrak{u}_{0}$ such that $\mathrm{u}_{1} \in \mathrm{E} \backslash \Omega$ and $\inf _{\partial \Omega} \varphi>\max \left\{\varphi\left(u_{0}\right), \varphi\left(u_{1}\right)\right\}$. Let

$$
\Gamma=\left\{\mathrm{g} \in \mathrm{C}([0,1], \mathrm{E}): \mathrm{g}(0)=\mathrm{u}_{0}, \mathrm{~g}(1)=\mathrm{u}_{1}\right\} \quad \text { and } \quad \mathrm{c}=\inf _{\mathrm{g} \in \Gamma \mathrm{max} \in[0,1]} \varphi(\mathrm{g}(\mathrm{s})) .
$$

If $\varphi$ satisfies the (PS $)_{c}$-condition, then $c$ is a critical value of $\varphi$ and $c>\max \left\{\varphi\left(u_{0}\right), \varphi\left(u_{1}\right)\right\}$.

To follow the ideas of the variational approach for impulsive differential equations of $[10,16]$, for each $v \in \mathrm{H}_{0}^{1}(0, \mathrm{~T})$, we have

$$
\begin{aligned}
\int_{0}^{T} u^{\prime \prime}(t) v(t) d t & =\int_{0}^{t_{1}} u^{\prime \prime}(t) v(t) d t+\sum_{i=1}^{N} \int_{t_{i}}^{s_{i}} u^{\prime \prime}(t) v(t) d t+\sum_{i=1}^{N-1} \int_{s_{i}}^{t_{i+1}} u^{\prime \prime}(t) v(t) d t+\int_{s_{N}}^{T} u^{\prime \prime}(t) v(t) d t \\
& =-\int_{0}^{T} u^{\prime}(t) v^{\prime}(t) d t+\sum_{i=1}^{N}\left[u^{\prime}\left(t_{i}^{-}\right)-u^{\prime}\left(t_{i}^{+}\right)\right] v\left(t_{i}\right)+\sum_{i=1}^{N}\left[u^{\prime}\left(s_{i}^{-}\right)-u^{\prime}\left(s_{i}^{+}\right)\right] v\left(s_{i}\right),
\end{aligned}
$$


which combined with (1.1) yields that

$$
\begin{aligned}
\int_{0}^{T} u^{\prime \prime}(t) v(t) d t= & -\int_{0}^{T} u^{\prime}(t) v^{\prime}(t) d t+\sum_{i=1}^{N}\left[\alpha_{i-1}-\alpha_{i}\right] v\left(t_{i}\right) \\
& -\sum_{i=0}^{N-1}\left(\int_{s_{i}}^{t_{i+1}} D_{x} F_{i}\left(t, u(t)-u\left(t_{i+1}\right)\right) d t\right) v\left(t_{i+1}\right) .
\end{aligned}
$$

On the other hand,

$$
\begin{aligned}
\int_{0}^{T} u^{\prime \prime}(t) v(t) d t & =-\sum_{i=0}^{N} \int_{s_{i}}^{t_{i+1}} D_{x} F_{i}\left(t, u(t)-u\left(t_{i+1}\right)\right) v(t) d t+\sum_{i=1}^{N} \int_{t_{i}}^{s_{i}} \frac{d}{d t}\left[\alpha_{i}\right] v(t) d t \\
& =-\sum_{i=0}^{N} \int_{s_{i}}^{t_{i+1}} D_{x} F_{i}\left(t, u(t)-u\left(t_{i+1}\right)\right) v(t) d t .
\end{aligned}
$$

Thus, in view of $v\left(t_{\mathrm{N}+1}\right)=v(\mathrm{~T})=0,(2.3)$, and (2.4), we find that

$$
-\int_{0}^{T} u^{\prime}(t) v^{\prime}(t) d t+\sum_{i=1}^{N}\left[\alpha_{i-1}-\alpha_{i}\right] v\left(t_{i}\right)=-\sum_{i=0}^{N} \int_{s_{i}}^{t_{i+1}} D_{x} F_{i}\left(t, u(t)-u\left(t_{i+1}\right)\right)\left(v(t)-v\left(t_{i+1}\right)\right) d t .
$$

Considering the aforementioned equality, we introduce the following concept of weak solution for (1.1).

Definition 2.3. A function $u \in H_{0}^{1}(0, T)$ is a weak solution of (1.1) if (2.5) holds for any $v \in H_{0}^{1}$.

Consider the functional $\Phi: \mathrm{H}_{0}^{1} \rightarrow \mathbb{R}$ defined by

$$
\Phi(u):=\frac{1}{2} \int_{0}^{T}\left|u^{\prime}(t)\right|^{2} d t-\sum_{i=1}^{N}\left(\alpha_{i-1}-\alpha_{i}\right) u\left(t_{i}\right)-\sum_{i=0}^{N} \varphi_{i}(u),
$$

where

$$
\varphi_{i}(u):=\int_{s_{i}}^{t_{i+1}} F_{i}\left(t, u(t)-u\left(t_{i+1}\right)\right) d t .
$$

For $u$ and $v$ fixed in $H_{0}^{1}(0, T)$ and $\lambda \in[-1,1]$, it follows from (2.2) that

$$
\left|u(t)-u\left(t_{i+1}\right)\right| \leqslant 2\|u\|_{\infty} \leqslant 2 \beta\|u\|,
$$

which combined with assumption (A) yields that $\varphi_{i}(u)$ is well-defined on $H_{0}^{1}(0, T)$. Using assumption (A) again, we see that

$$
\begin{gathered}
\lim _{\lambda \rightarrow 0} \frac{1}{\lambda}\left[F_{i}\left(t, u(t)-u\left(t_{i+1}\right)+\lambda v(t)-\lambda v\left(t_{i+1}\right)\right)-F_{i}\left(t, u(t)-u\left(t_{i+1}\right)\right)\right] \\
=D_{x} F_{i}\left(t, u(t)-u\left(t_{i+1}\right)\right)\left(v(t)-v\left(t_{i+1}\right)\right), \quad \text { for a.e. } t \in\left(s_{i}, t_{i+1}\right] .
\end{gathered}
$$

It follows from (2.6) that

$$
\left|u(t)-u\left(t_{i+1}\right)+\lambda \theta\left(v(t)-v\left(t_{i+1}\right)\right)\right| \leqslant 2 \beta(\|u\|+\|v\|), \quad \text { for } \theta \in(0,1),
$$

which combined with mean value theorem and assumption (A) yields to

$$
\left|\frac{1}{\lambda}\left[F_{i}\left(t, u(t)-u\left(t_{i+1}\right)+\lambda\left(v(t)-v\left(t_{i+1}\right)\right)\right)-F_{i}\left(t, u(t)-u\left(t_{i+1}\right)\right)\right]\right|
$$




$$
\begin{aligned}
& =\left|D_{x} F_{i}\left(t, u(t)-u\left(t_{i+1}\right)+\lambda \theta\left(v(t)-v\left(t_{i+1}\right)\right)\right)\left(v(t)-v\left(t_{i+1}\right)\right)\right| \\
& \leqslant \max _{z \in[0,2 \beta(\|u\|+\|v\|)]} a(z) 2 \beta\|v\| b(t) \in L^{1}\left(s_{i}, t_{i+1} ; \mathbb{R}^{+}\right),
\end{aligned}
$$

for some $\theta \in(0,1)$. Lebesgue's dominated convergence theorem shows that $\varphi_{i}$ has at every point $u$ a directional derivative

$$
\left\langle\varphi_{i}^{\prime}(u), v\right\rangle=\int_{s_{i}}^{t_{i+1}} D_{x} F_{i}\left(t, u(t)-u\left(t_{i+1}\right)\right)\left(v(t)-v\left(t_{i+1}\right)\right) d t .
$$

Moreover, in view of assumption (A) and (2.6), we have

$$
\left|\left\langle\varphi_{i}^{\prime}(u), v\right\rangle\right| \leqslant \int_{s_{i}}^{t_{i+1}}\left|D_{x} F_{i}\left(t, u(t)-u\left(t_{i+1}\right)\right)\left\|\left(v(t)-v\left(t_{i+1}\right)\right) \mid d t \leqslant 2 \beta \int_{s_{i}}^{t_{i+1}} b(t) d t \max _{z \in[0,2 \beta\|u\|]} a(z)\right\| v \| .\right.
$$

Thus $\varphi_{i}^{\prime}(u) \in\left(H_{0}^{1}(0, T)\right)^{*}$. Suppose $u_{n} \rightarrow u$ in $H_{0}^{1}(0, T)$, then $\left\{u_{n}\right\}$ converges uniformly to $u$ on $[0, T]$ by (2.2). Furthermore, it follows from (2.7) that

$$
\left\|\varphi_{i}^{\prime}\left(u_{n}\right)-\varphi_{i}^{\prime}(u)\right\| \leqslant 2 \beta \int_{s_{i}}^{t_{i+1}}\left|D_{x} F_{i}\left(t, u_{n}(t)-u_{n}\left(t_{i+1}\right)\right)-D_{x} F_{i}\left(t, u(t)-u\left(t_{i+1}\right)\right)\right| d t .
$$

Thus $\varphi_{i}^{\prime}$ is continuous from $\mathrm{H}_{0}^{1}(0, \mathrm{~T})$ into $\left(\mathrm{H}_{0}^{1}(0, \mathrm{~T})\right)^{*}$. So $\Phi \in \mathrm{C}^{1}\left(\mathrm{H}_{0}^{1}(0, \mathrm{~T}), \mathbb{R}\right)$ and

$$
\left\langle\Phi^{\prime}(u), v\right\rangle=\int_{0}^{T} u^{\prime}(t) v^{\prime}(t) d t-\sum_{i=1}^{N}\left(\alpha_{i-1}-\alpha_{i}\right) v\left(t_{i}\right)-\sum_{i=0}^{N} \int_{s_{i}}^{t_{i+1}} D_{x} F_{i}\left(t, u(t)-u\left(t_{i+1}\right)\right)\left(v(t)-v\left(t_{i+1}\right)\right) d t
$$

Then critical points of $\Phi$ correspond to weak solutions of the problem (1.1).

Lemma 2.4. If assumption $(H)$ holds, then for each $i=0,1, \cdots, N$ there exist $M_{i}, m_{i}, b_{i} \in L^{1}\left(s_{i}, t_{i+1}\right)$ which are almost everywhere positive such that

$$
F_{i}(t, x) \leqslant M_{i}(t)|x|^{\sigma_{i}}, \text { for a.e. } t \in\left(s_{i}, t_{i+1}\right] \text { and }|x| \leqslant 1
$$

and

$$
F_{i}(t, x) \geqslant m_{i}(t)|x|^{\sigma_{i}}-b_{i}(t), \quad \text { for a.e. } t \in\left(s_{i}, t_{i+1}\right] \text { and } x \in \mathbb{R} \text {, }
$$

where

$$
m_{i}(t):=\min _{|x|=1} F_{i}(t, x) \text { and } \quad M_{i}(t):=\max _{|x|=1} F_{i}(t, x), \quad \text { for a.e. } t \in\left(s_{i}, t_{i+1}\right] .
$$

Proof. It follows from assumption $(\mathrm{H})$ that

$$
0<m_{i}(t) \leqslant F_{i}\left(t, \frac{x}{|x|}\right) \leqslant M_{i}(t), \quad \text { for a.e. } t \in\left(s_{i}, t_{i+1}\right]
$$

and

$$
M_{i}(t) \leqslant \max _{|x| \leqslant 1} F_{i}(t, x) \leqslant \max _{|x| \leqslant 1} a(|x|) b(t) \in L^{1}\left(s_{i}, t_{i+1}\right) .
$$

For each $i=0,1, \cdots, N$, define $T_{i}:(0,+\infty) \rightarrow \mathbb{R}$ by

$$
\mathrm{T}_{i}(z)=\mathrm{F}_{i}\left(\mathrm{t}, \frac{x}{z}\right) z^{\sigma_{i}}, \quad \text { for a.e. } \mathrm{t} \in\left(\mathrm{s}_{i}, \mathrm{t}_{i+1}\right] \text { and } x \neq 0 .
$$

Condition $(H)$ implies that $T_{i}$ is nonincreasing. Thus, for a.e. $t \in\left(s_{i}, t_{i+1}\right]$, we have

$$
F_{i}(t, x) \leqslant F_{i}\left(t, \frac{x}{|x|}\right)|x|^{\sigma_{i}}, \quad \text { if } 0<|x| \leqslant 1
$$


and

$$
F_{i}(t, x) \geqslant F_{i}\left(t, \frac{x}{|x|}\right)|x|^{\sigma_{i}}, \quad \text { if }|x| \geqslant 1 .
$$

So (2.8) holds. Moreover, for a.e. $t \in\left(s_{i}, t_{i+1}\right]$ and $|x| \leqslant 1$, we have

$$
\left.\left|F_{i}(t, x)-m_{i}(t)\right| x\right|^{\sigma_{i}} \mid \leqslant \max _{|x| \leqslant 1} a(|x|) b(t)+m_{i}(t):=b_{i}(t),
$$

which combined with (2.10) yields (2.9).

\section{Proof of Theorem 1.1}

Proof. We complete the proof in four steps.

Step 1. $\Phi(\mathfrak{u})$ satisfies PS condition on $\mathrm{H}_{0}^{1}(0, T)$, that is, every sequence $\left\{\mathfrak{u}_{k}\right\}$ in $\mathrm{H}_{0}^{1}(0, T)$ such that $\Phi\left(\mathfrak{u}_{k}\right)$ is bounded and $\Phi^{\prime}\left(u_{k}\right) \rightarrow 0$ as $k \rightarrow+\infty$ contains a convergent subsequence. It is clear that

$$
\left|\sum_{i=1}^{N}\left(\alpha_{i-1}-\alpha_{i}\right) u\left(t_{i}\right)\right| \leqslant \sum_{i=1}^{N}\left|\alpha_{i-1}-\alpha_{i}\right|\|u\|_{\infty} \leqslant \sum_{i=1}^{N}\left|\alpha_{i-1}-\alpha_{i}\right| \beta\|u\| .
$$

Let $\sigma:=\min \left\{\sigma_{i}: i=0,1, \cdots, N\right\}$, then $\sigma>2$. By $(\mathrm{H})$ and (3.1), we have

$$
\begin{aligned}
\sigma \Phi\left(u_{k}\right)-\left\langle\Phi^{\prime}\left(u_{k}\right), u_{k}\right\rangle= & \left(\frac{\sigma}{2}-1\right) \int_{0}^{T}\left|u_{k}^{\prime}(t)\right|^{2} d t-(\sigma-1) \sum_{i=1}^{N}\left(\alpha_{i-1}-\alpha_{i}\right) u_{k}\left(t_{i}\right) \\
& -\sum_{i=0}^{N} \int_{s_{i}}^{t_{i+1}} \sigma F_{i}\left(t, u_{k}(t)-u_{k}\left(t_{i+1}\right)\right)-D_{x} F_{i}\left(t, u_{k}(t)-u_{k}\left(t_{i+1}\right)\right) \\
& \times\left(u_{k}(t)-u_{k}\left(t_{i+1}\right)\right) d t \\
\geqslant & \left(\frac{\sigma}{2}-1\right)\left\|u_{k}\right\|^{2}-(\sigma-1) \beta \sum_{i=1}^{N}\left|\alpha_{i-1}-\alpha_{i}\right|\left\|u_{k}\right\|
\end{aligned}
$$

which implies that $\left\{u_{k}\right\}$ is bounded in $H_{0}^{1}(0, T)$. Since $H_{0}^{1}(0, T)$ is a reflexive Banach space, passing to a subsequence if necessary, we may assume that there is a $u_{0} \in H_{0}^{1}(0, T)$ such that $u_{k} \rightarrow u_{0}$ in $H_{0}^{1}(0, T)$. Then $\left\{u_{k}\right\}$ converges uniformly to $u_{0}$ on $[0, T]$ and $u_{k} \rightarrow u_{0}$ in $L^{2}(0, T)$. Notice that

$$
\begin{aligned}
& \left\langle\Phi^{\prime}\left(u_{m}\right)-\Phi^{\prime}\left(u_{n}\right), u_{m}-u_{n}\right\rangle \\
& =\int_{0}^{T}\left|u_{m}^{\prime}(t)-u_{n}^{\prime}(t)\right|^{2} d t-\sum_{i=0}^{N} \int_{s_{i}}^{t_{i+1}}\left[D_{x} F_{i}\left(t, u_{m}(t)-u_{m}\left(t_{i+1}\right)\right)\right. \\
& \left.\quad-D_{x} F_{i}\left(t, u_{n}(t)-u_{n}\left(t_{i+1}\right)\right)\right]\left[u_{m}(t)-u_{m}\left(t_{i+1}\right)-u_{n}(t)+u_{n}\left(t_{i+1}\right)\right] d t .
\end{aligned}
$$

In view of assumption $(\mathrm{A})$ and

$$
\begin{aligned}
\left|u_{m}(t)-u_{m}\left(t_{i+1}\right)-u_{n}(t)+u_{n}\left(t_{i+1}\right)\right| & \leqslant\left|u_{m}(t)-u_{n}(t)\right|+\left|u_{m}\left(t_{i+1}\right)-u_{n}\left(t_{i+1}\right)\right| \\
& \leqslant 2\left\|u_{m}-u_{n}\right\|_{\infty} \rightarrow 0, \quad \text { as } m, n \rightarrow \infty,
\end{aligned}
$$

we have that the second term on the right hand of (3.3) converges to 0 as $m, n \rightarrow \infty$. What is more, the fact that $\Phi^{\prime}\left(u_{k}\right) \rightarrow 0$ as $k \rightarrow+\infty$ implies

$$
\left|\left\langle\Phi^{\prime}\left(u_{m}\right)-\Phi^{\prime}\left(u_{n}\right), u_{m}-u_{n}\right\rangle\right| \leqslant\left\|\Phi^{\prime}\left(u_{m}\right)-\Phi^{\prime}\left(u_{n}\right)\right\|\left\|u_{m}-u_{n}\right\| \rightarrow 0,
$$

as $m, n \rightarrow \infty$. Consequently, $\left\|u_{m}-u_{n}\right\| \rightarrow 0$ as $m, n \rightarrow \infty$. By the completeness of $H_{0}^{1}(0, T)$, we know that $\left\{u_{k}\right\}$ possesses a convergent subsequence in $\mathrm{H}_{0}^{1}(0, T)$. 
Step 2. $\Phi(u)$ has mountain pass geometric structure.

Let $B_{r}$ be the open ball in $H_{0}^{1}(0, T)$ with radius $r$ and centered at 0 and let $\partial B_{r}$ and $\overline{B_{r}}$ denote the boundary and closure of $B_{r}$, respectively. For each $u \in \partial B_{(2 \beta)^{-1}}$, where $\beta$ is listed in (2.2), we have

$$
\left|u(t)-u\left(t_{i+1}\right)\right| \leqslant 2\|u\|_{\infty} \leqslant 2 \beta\|u\|=1 .
$$

Thus, using (2.8), we find that, for each $u \in \partial \mathrm{B}_{(2 \beta)^{-1}}$,

$$
\int_{s_{i}}^{t_{i+1}} F_{i}\left(t, u(t)-u\left(t_{i+1}\right)\right) d t \leqslant \int_{s_{i}}^{t_{i+1}} M_{i}(t)\left|u(t)-u\left(t_{i+1}\right)\right|^{\sigma_{i}} d t \leqslant \int_{s_{i}}^{t_{i+1}} M_{i}(t) d t
$$

which combined with (3.1) yields that, for any $u \in \partial B_{(2 \beta)^{-1}}$,

$$
\Phi(u) \geqslant \frac{1}{2}\left[\frac{1}{4 \beta^{2}}-\sum_{i=1}^{N}\left|\alpha_{i-1}-\alpha_{i}\right|-2 \sum_{i=0}^{N} \int_{s_{i}}^{t_{i+1}} M_{i}(t) d t\right]:=\gamma .
$$

In view of (1.2), we have $\Phi(u) \geqslant \gamma>0=\Phi(0)$ for any $u \in \partial B_{(2 \beta)^{-1}}$. Thus

$$
\inf _{u \in \partial B_{(2 \beta)^{-1}}} \Phi(u)>\Phi(0) .
$$

Let $\xi>0$ and $w \in \mathrm{H}_{0}^{1}(0, \mathrm{~T})$ with $\|w\|=1$ and $w(\mathrm{t})$ is not a constant for a.e. $\left[0, \mathrm{t}_{1}\right]$. It follows from (2.9) that

$$
\int_{s_{i}}^{t_{i+1}} F_{i}\left(t,\left(w(t)-w\left(t_{i+1}\right)\right) \xi\right) d t \geqslant\left(\int_{s_{i}}^{t_{i+1}} m_{i}(t)\left|w(t)-w\left(t_{i+1}\right)\right|^{\sigma_{i}} d t\right) \xi^{\sigma_{i}}-\int_{s_{i}}^{t_{i+1}} b_{i}(t) d t .
$$

Let $Q_{i}:=\int_{s_{i}}^{t_{i+1}} m_{i}(t)\left|w(t)-w\left(t_{i+1}\right)\right|^{\sigma_{i}} d t$. Then we have that

$$
0 \leqslant Q_{i} \leqslant(2 \beta)^{\sigma_{i}} \int_{s_{i}}^{t_{i+1}} m_{i}(t) d t \text { and } Q_{0}>0
$$

In fact, suppose that $\int_{0}^{t_{1}} m_{0}(t)\left|w(t)-w\left(t_{1}\right)\right|^{\sigma_{0}} d t=0$, since $m_{0}(t)$ is almost everywhere positive, we have $w(t)=w\left(t_{1}\right)$ for a.e. $\left[0, t_{1}\right]$, which is a contradiction to the assumption on $w$. In view of (3.4), we have

$$
\begin{aligned}
\Phi(\xi w) & =\frac{1}{2} \xi^{2}-\sum_{i=1}^{N}\left(\alpha_{i-1}-\alpha_{i}\right) w\left(t_{i}\right) \xi-\sum_{i=0}^{N} \int_{s_{i}}^{t_{i+1}} F_{i}\left(t,\left(w(t)-w\left(t_{i+1}\right)\right) \xi\right) d t \\
& \leqslant \frac{1}{2} \xi^{2}+\sum_{i=1}^{N}\left|\alpha_{i-1}-\alpha_{i}\right| \beta \xi-\sum_{i=0}^{N} Q_{i} \xi^{\sigma_{i}}+\sum_{i=0}^{N} \int_{s_{i}}^{t_{i+1}} b_{i}(t) d t .
\end{aligned}
$$

Since $\sigma_{i}>2$, we have $\Phi(\xi w) \rightarrow-\infty$ as $\xi \rightarrow+\infty$. Thus, there exists $\xi_{0}$ with $\left\|\xi_{0} w\right\|>(2 \beta)^{-1}$ such that

$$
\inf _{u \in \partial B_{(2 \beta)^{-1}}} \Phi(u)>\Phi\left(\xi_{0} w\right) .
$$

Therefore it follows from Steps 1-2 and Lemma 2.2 that there exists $u_{1}^{*} \in H_{0}^{1}(0, T)$ such that $\Phi^{\prime}\left(u_{1}^{*}\right)=0$ with

$$
\Phi\left(u_{1}^{*}\right)>\max \left\{\Phi(0), \Phi\left(\xi_{0} w\right)\right\} \geqslant \Phi(0)=0,
$$

so $u_{1}^{*}$ is a nonzero weak solution of problem (1.1).

Step 3. If $\alpha_{j-1} \neq \alpha_{j}$ for some $j=1,2, \cdots, N$, then $\Phi(u)$ has a nonzero local minimum $u_{2}^{*}$ in $\overline{B_{(2 \beta)^{-1}}}$, where $\beta$ is listed in (2.2). 
Since $\overline{\mathrm{B}_{(2 \beta)^{-1}}}$ is a closed convex set, $\overline{\mathrm{B}_{(2 \beta)^{-1}}}$ is weak sequentially closed. Furthermore, $\Phi(\mathrm{u})$ is sequentially weakly lower semi-continuous on $\mathrm{H}_{0}^{1}(0, \mathrm{~T})$ as the sum of a convex continuous function and of a weakly continuous one. Thus it follows from Lemma 2.1 that there exists a $u_{2}^{*} \in \overline{\mathrm{B}_{(2 \beta)^{-1}}}$ such that

$$
\Phi\left(u_{2}^{*}\right)=\frac{\min }{\mathrm{B}_{(2 \beta)^{-1}}} \Phi(\mathrm{u}) .
$$

What is more, $u_{2}^{*} \neq 0$. In fact, since $F_{i}(t, 0)=0$ for a.e. $t \in\left(s_{i}, t_{i+1}\right]$, we find that $F_{i}(t, x) \geqslant 0$ for a.e. $\mathrm{t} \in\left(s_{i}, t_{i+1}\right]$ and $x \in \mathbb{R}$ by $(\mathrm{H})$. Thus $\varphi_{i} \geqslant 0$. Let

$$
u^{\sharp}(t):= \begin{cases}\alpha_{j-1}-\alpha_{j}, & \text { if } t=t_{j}, \\ 0, & \text { if } t \in[0, T] \text { and } t \neq t_{j},\end{cases}
$$

then $u^{\sharp} \in \overline{\mathrm{B}_{(2 \beta)^{-1}}}$ and $\Phi\left(u^{\sharp}\right) \leqslant-\left(\alpha_{j-1}-\alpha_{j}\right)^{2}<0$. So

$$
\Phi\left(u_{2}^{*}\right) \leqslant \Phi\left(u^{\sharp}\right)<0
$$

and the assertion follows.

Step 4. $u_{1}^{*}$ and $u_{2}^{*}$ are different and both bounded.

In view of (3.6) and (3.7), we have

$$
\Phi\left(u_{1}^{*}\right)>0>\Phi\left(u_{2}^{*}\right),
$$

so $u_{1}^{*}$ and $u_{2}^{*}$ are different. From the inf max characterization of $u_{1}^{*}$ in Lemma 2.2 and (3.5), we find that

$$
\Phi\left(u_{1}^{*}\right)=\inf _{g \in \Gamma} \max _{s \in[0,1]} \Phi(g(s)) \leqslant \max _{s \in[0,1]} \Phi\left(\xi_{0} w s\right) \leqslant \max _{s \in[0,1]} h(s),
$$

where

$$
h(s):=\frac{\xi_{0}^{2} s^{2}}{2}+\sum_{i=1}^{N}\left|\alpha_{i-1}-\alpha_{i}\right| \beta \xi_{0} s-\sum_{i=0}^{N} Q_{i} \xi_{0}^{\sigma_{i}} s^{\sigma_{i}}+\sum_{i=0}^{N} \int_{s_{i}}^{t_{i+1}} b_{i}(t) d t .
$$

Since $h(s)$ is continuous on $[0,1]$, we have $\Phi\left(u_{1}^{*}\right)$ is bounded above and so is $\Phi\left(u_{2}^{*}\right)$ by (3.8). Similar as (3.2), for $\mathrm{u}^{*} \in \mathrm{H}_{0}^{1}(0, \mathrm{~T})$, we get

$$
\left(\frac{\sigma}{2}-1\right)\left\|u^{*}\right\|^{2}-(\sigma-1) \beta \sum_{i=1}^{N}\left|\alpha_{i-1}-\alpha_{i}\right|\left\|u^{*}\right\| \leqslant \sigma \Phi\left(u^{*}\right)-\left\langle\Phi^{\prime}\left(u^{*}\right), u^{*}\right\rangle .
$$

Since $u_{1}^{*}$ and $u_{2}^{*}$ are both critical points of $\Phi$, furthermore $\Phi\left(u_{1}^{*}\right)$ and $\Phi\left(u_{2}^{*}\right)$ are both bounded above, (3.9) implies that $u_{1}^{*}$ and $u_{2}^{*}$ are both bounded in $\mathrm{H}_{0}^{1}(0, T)$. This completes the proof.

\section{Acknowledgment}

This work has been partially supported by National Natural Science Foundation of China (No.11401420, No.11326117, No.11626182), SXNSF (No.2013021001-2, No.201601D102002), YFTUT (No.2013T062), TFTUT (No.tyut-rc201212a), the Ministerio de Economia y Competitividad of Spain under Grant MTM201675140-P of the AEI and co-financed by European Community fund FEDER and XUNTA de Galicia under grants GRC2015-004 and R2016/022.

\section{References}

[1] G. A. Afrouzi, A. Hadjian, V. D. Rădulescu, Variational approach to fourth-order impulsive differential equations with two control parameters, Results Math., 65 (2014), 371-384. 1

[2] G. A. Afrouzi, A. Hadjian, S. Shokooh, Infinitely many solutions for a Dirichlet boundary value problem with impulsive condition, Politehn. Univ. Bucharest Sci. Bull. Ser. A Appl. Math. Phys., 77 (2015), 9-22. 1

[3] L. Bai, J. J. Nieto, Variational approach to differential equations with not instantaneous impulses, Appl. Math. Lett., (2017), (In press). 1 
[4] G. Bonanno, R. Rodríguez-López, S. Tersian, Existence of solutions to boundary value problem for impulsive fractional differential equations, Fract. Calc. Appl. Anal., 17 (2014), 717-744. 1

[5] V. Colao, L. Muglia, H.-K. Xu, Existence of solutions for a second-order differential equation with non-instantaneous impulses and delay, Ann. Mat. Pura Appl., 195 (2016), 697-716. 1

[6] B.-X. Dai, D. Zhang, The existence and multiplicity of solutions for second-order impulsive differential equations on the half-line, Results Math., 63 (2013), 135-149. 1

[7] E. Hernández, D. O’Regan, On a new class of abstract impulsive differential equations, Proc. Amer. Math. Soc., 141 (2013), 1641-1649. 1

[8] J. Mawhin, M. Willem, Critical point theory and Hamiltonian systems, Applied Mathematical Sciences, SpringerVerlag, New York, (1989). 2.2

[9] J. J. Nieto, Variational formulation of a damped Dirichlet impulsive problem, Appl. Math. Lett., 23 (2010), 940-942. 1

[10] J. J. Nieto, D. O’Regan, ariational approach to impulsive differential equations, Nonlinear Anal. Real World Appl., 10 (2009), 680-690. 1, 2

[11] M. Pierri, H. R. Henríquez, A. Prokopczyk, Global solutions for abstract differential equations with non-instantaneous impulses, Mediterr. J. Math., 13 (2016), 1685-1708. 1

[12] M. Pierri, D. O'Regan, V. Rolnik, Existence of solutions for semi-linear abstract differential equations with not instantaneous impulses, Appl. Math. Comput., 219 (2013), 6743-6749. 1

[13] R. Rodríguez-López, S. Tersian, Multiple solutions to boundary value problem for impulsive fractional differential equations, Fract. Calc. Appl. Anal., 17 (2014), 1016-1038. 1

[14] A. M. Samoilenko, N. A. Perestyuk, Impulsive differential equations, With a preface by Yu. A. Mitropolskiľand a supplement by S. I. Trofimchuk, Translated from the Russian by Y. Chapovsky, World Scientific Series on Nonlinear Science, Series A: Monographs and Treatises, World Scientific Publishing Co., Inc., River Edge, NJ, (1995).

[15] J.-T. Sun, H.-B. Chen, Multiplicity of solutions for a class of impulsive differential equations with Dirichlet boundary conditions via variant fountain theorems, Nonlinear Anal. Real World Appl., 11 (2010), 4062-4071.

[16] Y. Tian, W.-G. Ge, Applications of variational methods to boundary-value problem for impulsive differential equations, Proc. Edinb. Math. Soc., 51 (2008), 509-528. 1, 2

[17] E. Zeidler, Nonlinear functional analysis and its applications, III, Variational methods and optimization, Translated from the German by Leo F. Boron, Springer-Verlag, New York, (1985). 2.1 